\title{
Factores de riesgo de la precocidad sexual en adolescentes
}

\author{
P. Royuela Ruiz ${ }^{a}$, L. Rodríguez Molinero ${ }^{b}$, J. M. Marugán de Miguelsanz ${ }^{c}$, \\ V. Carbajosa Rodríguez ${ }^{d}$
}

Publicado en Internet:

18-junio-2015

Pablo Royuela Ruiz: pabloroyuela@yahoo.es

\begin{abstract}
aMédico de Familia. Unidad de Urgencias Hospital Universitario Río Hortega. Valladolid. España - 'Pediatra. Centro de Salud Casa del Barco. Valladolid. España • 'Servicio de Pediatría. Hospital Clínico Universitario. Valladolid. España • dMédico de Familia. Unidad de Urgencias Hospital Universitario Río Hortega. Valladolid. España.
\end{abstract}

Introducción: en los últimos años parece que la precocidad sexual de nuestros jóvenes ha ido en aumento. En este estudio queremos averiguar cuáles son los factores de riesgo adolescente que se relacionan de forma estadísticamente significativa con tener relaciones sexuales completas.

Material y métodos: encuesta a 1289 adolescentes de segundo y tercero de Educación Secundaria Obligatoria de Castilla y León (España) en el curso 2010-2011. Analizamos múltiples variables epidemiológicas, calculando medidas de centralización y dispersión en las variables cuantitativas, y de distribución de frecuencias en las cualitativas.

Resultados: hemos estudiado a 563 varones y 726 mujeres. La edad media de los encuestados fue de 14,05 años (mediana y moda: 14 años; rango: 12-17 años), sin existir diferencias entre sexos. El 17,5\% había tenido relaciones sexuales con penetración (el $9 \%$ a los $12 ;$ el $6 \%$ a los 13 ; el $10,6 \%$ a los 14 ; el $34,2 \%$ a los 15 ; el $53,2 \%$ a los 16 y el $57,1 \%$ a los 17 años). Este porcentaje era mayor en los chicos $(20,2 \%)$ que en las chicas $(15,4 \%)$. Otros factores asociados de forma estadísticamente significativa $(p<0,05)$ con haber tenido relaciones sexuales coitales fueron mayor edad, peor relación con sus padres, padres divorciados, peor relación entre sus padres, fumar, beber alcohol, emborracharse, consumir drogas, peor salud actual, peor alimentación, peor memoria, ser más erotofílicos y definirse como agnóstico o ateo.

Palabras clave:

- Sexualidad

- Adolescencia

- Factores de riesgo
Conclusiones: además de con el alcohol, el tabaco y otras drogas también es factor de riesgo de precocidad sexual adolescente tener una mala relación con los padres y de los padres entre sí.

\section{Risk factors of sexual precocity in adolescents}

Introduction: in the last years, sexual initiation in our young people seems to have anticipated. In this study we want to find out what risk factors have statistically significant relation to having sexual relations with penetration in adolescents.

Methods: 1289 students of $2 .^{\circ}$ and $3 .^{\circ}$ grade of Secondary Education in the region of Castilla-León (Spain) were interrogated in the 2010-2011 school year. Multiple epidemiological variables were analyzed, calculating centralization and dispersion in quantitative variables, and distribution of frequencies in qualitative variables.

Results: 563 boys and 726 girls were studied. The students' media age was 14.05 years (median and mode: 14 years; range: $12-17$ years). $17.5 \%$ had had sexual relations with penetration. ( $9 \%$ at $12 ; 6 \%$ at $13 ; 10.6 \%$ at $14 ; 34.2 \%$ at $15 ; 53.2 \%$ at 16 and $57.1 \%$ at 17 years). This percentage was greater in boys $(20.2 \%)$ than girls $(15.4 \%)$. Other statistically significant associated factors $(p<0.05)$ were being older, worse relation with his parents, divorced parents, worse relation between their parents, smoking, drinking alcohol, getting drunk, consuming drugs, worse present health, worse feeding,

Key words: worse memory, having more erotophilia and being agnostic or atheist.

- Sexuality

- Adolescence

Conclusions: adolescent sexual precocious initiation risk factors are alcohol, tobacco, drug consump- Risk factors 


\section{INTRODUCCIÓN}

El inicio de las relaciones sexuales es muy variable entre las personas y, sin embargo, la precocidad y la promiscuidad de nuestros jóvenes parece que han ido en aumento ${ }^{1}$.

Sin entrar en prohibiciones morales o religiosas, el problema de la precocidad sexual es que se relaciona con otras prácticas sexuales de riesgo. Los adolescentes que se inician a edades muy tempranas refieren con mayor frecuencia tener una infección de transmisión sexual (ITS), un mayor número de parejas sexuales y un menor uso del preservativo ${ }^{2}$. Junto a todo lo anterior, el embarazo no deseado es otro riesgo relacionado con la precocidad sexual. El Observatorio de Salud Reproductiva del Consejo Superior de Investigaciones Científicas señala que, en los últimos años, se ha producido un aumento continuo del número de embarazos en la adolescencia, de manera que en la población de 15 a 19 años de edad la tasa de embarazos por mil mujeres ha pasado de 15,12 en 1999 a 24,21 en 2009. Algo similar ha ocurrido con la tasa de fecundidad para este grupo de edad, que en España era de 8,54 en 1999 y ascendió a 11,78 en 2009. Esta situación llevó aparejado un incremento en la proporción de embarazos no deseados, que pasaron del 95,35\% en 1999 al 98,15\% en 2009, y la tasa de interrupciones voluntarias del embarazo por cada mil mujeres de 15 a 19 años de edad pasó de 7,49 en el año 2000 a 12,74 en $2009^{3}$.

El propósito de este estudio es averiguar cuáles son los factores de riesgo para que un adolescente tenga relaciones sexuales completas en los estudiantes de 2.ำ y 3. de Educación Secundaria Obligatoria (ESO) de Castilla y León. Creemos que estos datos serán de gran utilidad para la promoción de la salud de los adolescentes actuales y futuros.

\section{MATERIAL Y MÉTODOS}

El grupo de población objeto de estudio son los alumnos matriculados en 2.․ y 3. de ESO en centros educativos de Castilla y León en el curso 2010-
2011. Forman un total de 44031 estudiantes (chicos: 50,84\%; chicas: $49,16 \%)^{4}$.

A la hora de recoger los datos, hemos utilizado el muestreo por conglomerados, seleccionando cada conglomerado de manera aleatoria. Los alumnos objeto de nuestro estudio se encuentran agrupados en una serie de centros educativos y cada curso (2.. y 3. de ESO) dentro de cada centro educativo es un conglomerado de alumnos ${ }^{5,6}$. Dentro de cada conglomerado seleccionado para la muestra se realizó la encuesta a todos los alumnos.

La muestra calculada a partir de la fórmula mostrada en la Fig. 1 debe ser posteriormente ponderada para evitar el llamado efecto del diseño, que es la proporción de la varianza del muestreo aleatorio simple explicada por la varianza del muestreo por conglomerados ${ }^{6}$.

Para saber la prevalencia esperada de relaciones sexuales coitales en chicas de 2.ํy y 3. ㅇ de ESO (p) de nuestro entorno hicimos primero una búsqueda bibliográfica, pero no encontramos ningún dato que nos fuera útil. Por eso, fue necesario recurrir a un estudio piloto en 40 adolescentes de $2 .^{\circ}$ y $3 . \circ$ de ESO de un centro educativo escogido al azar para poder calcular el tamaño muestral necesario y hacer más comprensible el cuestionario. En ese estudio piloto la prevalencia de relaciones coitales en

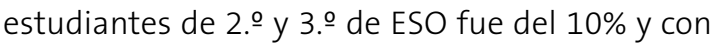
ese dato hicimos el cálculo del tamaño muestral.

A partir de la bibliografía consultada y de los datos obtenidos del estudio piloto, nos parece correcto cuantificar el efecto del diseño en 1,4; es decir, asumimos que hay un $40 \%$ de aumento de la varianza de los estimadores en nuestro diseño con respecto al muestreo aleatorio simple. Entonces, el tamaño muestral necesario utilizando muestreo

Figura 1. Fórmula empleada para calcular el tamaño muestral

$$
n=\frac{N \times Z_{1-a / 2}^{2} \times p \times q}{d^{2} \times(N-1)+Z_{1-a / 2}^{2} \times p \times q}
$$

d: error de estimación; $\mathbf{n}$ : tamaño de la muestra necesario; $\mathbf{N}$ : tamaño del universo de la población; $\mathbf{p}$ : prevalencia esperada; $\mathbf{q}=1-p$. 
por conglomerados, asumiendo un error del 3\% y con un nivel de confianza del $95 \%$ es de 528 chicas y de 529 varones.

Para llegar a conseguir ese número de encuestas realizadas, debemos llegar a un número suficiente de centros escolares. Así, de forma aleatoria, fuimos eligiendo centros escolares y recogiendo sus datos hasta tener más de 528 chicas y más de 529 chicos analizados. Cuando un centro escolar rehusaba participar, de nuevo elegíamos aleatoriamente otro centro escolar de la lista de los 390 centros que imparten ESO en Castilla y León.

Así, fueron necesarios 16 centros escolares para conseguir 1289 cuestionarios cumplimentados, correspondientes a 726 chicas y 563 chicos. Las encuestas se recogieron desde octubre del 2010 a abril del 2011 (Tabla 1).

El cuestionario constaba de 86 preguntas. El tiempo empleado era de unos 20 minutos. Las preguntas estaban agrupadas por capítulos según la temática relacionada: a) relación con sus padres; b) salud, deporte, autoestima, ideas religiosas; c) drogas legales e ilegales, y d) sexualidad.

Uno de los investigadores (además del tutor de los alumnos) estaba presente en el aula mientras se realizaba el cuestionario para explicar las dudas y asegurar el anonimato. Solo se pasaba la encuesta a los alumnos que hubieran traído la autorización paterna. Ningún progenitor se negó expresamente a que su hijo o hija participara en la encuesta, sin embargo, parece que por desidia o desinterés un $12 \%$ aproximado de los alumnos no trajo el permiso firmado por sus padres. A estos estudiantes no se les sometió al cuestionario y eran una gran mayoría

\begin{tabular}{|c|c|c|c|}
\hline \multicolumn{4}{|c|}{ Tabla 1. Edad y sexo de los encuestados } \\
\hline Edad & Chicos & Chicas & Total \\
\hline 12 & 14 & 9 & 23 \\
\hline 13 & 174 & 205 & 379 \\
\hline 14 & 221 & 300 & 521 \\
\hline 15 & 113 & 144 & 257 \\
\hline 16 & 35 & 59 & 94 \\
\hline 17 & 6 & 8 & 14 \\
\hline No contesta & & 1 & 1 \\
\hline Total & 563 & 726 & 1289 \\
\hline
\end{tabular}

Tabla 2. Encuestados que han tenido relaciones sexuales coitales según sexo (mayor precocidad en chicos, $p=0,027$ )

\begin{tabular}{|l|c|c|c|}
\hline & Chicos & Chicas & Total \\
\hline Sí & $114(20,2 \%)$ & $112(15,4 \%)$ & 226 \\
\hline No & $448(79,6 \%)$ & $609(83,9 \%)$ & 1057 \\
\hline No contesta & 1 & 5 & 6 \\
\hline Total & 563 & 726 & 1289 \\
\hline
\end{tabular}

de varones (razón fundamental de la diferencia de sexos encontrada en el análisis de los datos).

En el análisis estadístico se calculó la media, mediana, rango, desviación estándar y error estándar en el caso de las variables cuantitativas, y la distribución de frecuencias en las cualitativas. La comparación de medias se realizó por el test $t$ de Student, y de frecuencias por el test de Chi cuadrado, asumiendo una diferencia como significativa cuando la $p$ fue menor de 0,05. En el caso de variables dicotómicas también hemos calculado el riesgo relativo.

\section{RESULTADOS}

La edad media de los encuestados fue de 14,05 años con una desviación estándar de 0,98 (mediana y moda: 14 años; rango: 12-17 años). La edad media de los chicos era de 14,00 años (desviación estándar: 0,98) y de las chicas 14,09 años (desviación estándar: 0,97), no habiendo diferencias estadísticamente significativas.

El 17,5\% de los adolescentes de nuestra encuesta afirmaban haber tenido relaciones sexuales con penetración (Tablas 3 y 4). Este porcentaje era mayor en chicos $(20,2 \%)$ que en chicas $(15,4 \%)$. La diferencia entre chicos y chicas era estadísticamente significativa ( $p=0,027)$.

Como se ve en la Tabla 3, según avanza la edad de nuestros encuestados, cada vez más han tenido relaciones sexuales con penetración. Además, no hay que olvidar que los encuestados de 12 años son solo 23 y los de 17 años solo 14 , con lo que podemos entrar en sesgos si los comparamos con los otros grupos (mucho más numerosos). Por esta razón no los tendré en cuenta cuando ajuste los resultados por edad. 
Tabla 3. Encuestados que han tenido relaciones sexuales coitales según edad (mayor precocidad a mayor edad, $p<0,001$ )

\begin{tabular}{|l|c|c|c|c|c|c|c|c|}
\hline \multicolumn{1}{|c|}{ Edad } & $\mathbf{1 2}$ & $\mathbf{1 3}$ & $\mathbf{1 4}$ & $\mathbf{1 5}$ & $\mathbf{1 6}$ & $\mathbf{1 7}$ & No contesta & Total \\
\hline Sí & $2(9 \%)$ & $23(6 \%)$ & $55(10,6 \%)$ & $88(34,2 \%)$ & $50(53,2 \%)$ & $8(57,1 \%)$ & 226 \\
\hline No & $21(91 \%)$ & $355(94 \%)$ & $463(88,9 \%)$ & $168(65,4 \%)$ & $44(46,8 \%)$ & $5(35,7 \%)$ & 1 & 1057 \\
\hline No contesta & & 1 & 3 & 1 & & 1 & 6 \\
\hline Total & 23 & 379 & 521 & 257 & 94 & 14 & 1 & 1289 \\
\hline
\end{tabular}

\section{Relación con sus padres}

Globalmente, los que han tenido relaciones sexuales completas tienen una puntuación media de 7,45 puntos sobre 10 en la relación con sus padres, mientras que los que no las han tenido es de 8,34 $(p<0,001)$. Si tenemos en cuenta la edad de los encuestados, también se observa esta peor rela- ción de forma estadísticamente significativa en todos los grupos de edad (Tabla 4).

Además, la relación que tienen los padres de los adolescentes entre sí también es importante. Los adolescentes de 14 años con padres divorciados (Tabla 5) o con padres con una mala relación entre sí (Tabla 4) son más precoces.

\section{Tabla 4. Puntuaciones en diferentes escalas de los adolescentes encuestados}

\begin{tabular}{|c|c|c|c|c|}
\hline & Edad & $\begin{array}{l}\text { Sí relaciones } \\
\text { sexuales }\end{array}$ & $\begin{array}{l}\text { No relaciones } \\
\text { sexuales }\end{array}$ & Valor de $p$ \\
\hline \multirow{4}{*}{$\begin{array}{l}\text { Puntuación } \\
\text { relación con los padres } \\
\text { (del } 1 \text { al } 10)\end{array}$} & 13 & 7,61 & 8,57 & 0,049 \\
\hline & 14 & 7,47 & 8,28 & 0,02 \\
\hline & 15 & 7,57 & 8,07 & 0,03 \\
\hline & 16 & 7,14 & 7,98 & 0,046 \\
\hline \multirow{4}{*}{$\begin{array}{l}\text { Puntuación } \\
\text { relación entre los padres } \\
(\text { del } 1 \text { al } 10)\end{array}$} & 13 & 7,87 & 8,6 & $>0,05$ \\
\hline & 14 & 7,09 & 8,42 & 0,004 \\
\hline & 15 & 7,43 & 7,9 & $>0,05$ \\
\hline & 16 & 7,5 & 7,93 & $>0,05$ \\
\hline \multirow{4}{*}{$\begin{array}{l}\text { Salud actual } \\
\text { (del } 1 \text { al } 5)\end{array}$} & 13 & 4,48 & 4,59 & $>0,05$ \\
\hline & 14 & 4,41 & 4,53 & $>0,05$ \\
\hline & 15 & 4,16 & 4,41 & 0,026 \\
\hline & 16 & 3,98 & 4,49 & 0,006 \\
\hline \multirow{4}{*}{$\begin{array}{l}\text { Puntuación alimentación } \\
\text { (del } 1 \text { al 5) }\end{array}$} & 13 & 4,22 & 4,11 & $>0,05$ \\
\hline & 14 & 3,87 & 4,06 & $>0,05$ \\
\hline & 15 & 3,99 & 4,05 & $>0,05$ \\
\hline & 16 & 3,55 & 4,16 & 0,004 \\
\hline \multirow{4}{*}{$\begin{array}{l}\text { Puntuación memoria } \\
\text { (del } 1 \text { al 5) }\end{array}$} & 13 & 3,96 & 4,16 & $>0,05$ \\
\hline & 14 & 4,09 & 4,04 & $>0,05$ \\
\hline & 15 & 3,58 & 3,97 & 0,007 \\
\hline & 16 & 3,24 & 3,86 & 0,005 \\
\hline \multirow{4}{*}{$\begin{array}{l}\text { Puntuación erotofilia } \\
\text { (de } 0 \text { a 60) }\end{array}$} & 13 & 34,17 & 22,76 & $<0,001$ \\
\hline & 14 & 34,51 & 25,16 & $<0,001$ \\
\hline & 15 & 30,43 & 29,69 & $>0,05$ \\
\hline & 16 & 32,53 & 28,83 & $>0,05$ \\
\hline
\end{tabular}




\begin{tabular}{|l|c|l|c|c|}
\hline \multicolumn{1}{|c|}{ Factor de riesgo } & $\begin{array}{c}\text { Ajuste por } \\
\text { edad }\end{array}$ & $\begin{array}{c}\text { Porcentaje que han tenido } \\
\text { relaciones sexuales coitales }\end{array}$ & $\begin{array}{c}\text { Riesgo } \\
\text { relativo }\end{array}$ \\
\hline Chico frente a chica & 13 años & $10,9 \%$ frente a 1,96\% & 0,0003 & 5,57 \\
\hline Chico frente a chica & 14 años & $14,1 \%$ frente a $8 \%$ & 0,0275 & 1,75 \\
\hline $\begin{array}{l}\text { Padres divorciados frente a padres no } \\
\text { divorciados }\end{array}$ & 14 años & $19,1 \%$ frente a 9,6\% & 0,042 & 1,99 \\
\hline Fumar frente a no fumar & 13 años & $28 \%$ frente a 5\% & $<0,0001$ & 6,18 \\
\hline Fumar frente a no fumar & 14 años & $31 \%$ frente a $6 \%$ & $<0,001$ & $<0,0001$ \\
\hline Fumar frente a no fumar & 15 años & $59 \%$ frente a 20\% & $<0,0001$ & 2,54 \\
\hline Beber alcohol frente a no beber & 13 años & $15 \%$ frente a 1\% & $<0,0001$ & 11,73 \\
\hline Beber alcohol frente a no beber & 14 años & $11 \%$ frente a 2\% & $<0,0001$ & 4,71 \\
\hline Beber alcohol frente a no beber & 15 años & $60 \%$ frente a 18\% & $<0,0001$ & 2,16 \\
\hline Emborracharse frente a no emborracharse & 13 años & $24 \%$ frente a 3\% & $<0,0001$ & 6,97 \\
\hline Emborracharse frente a no emborracharse & 14 años & $27 \%$ frente a $5 \%$ & $<0,0001$ & 5,81 \\
\hline Emborracharse frente a no emborracharse & 15 años & $50 \%$ frente a 16\% & $<0,0001$ & 3,06 \\
\hline Consumir drogas frente a no consumo & Todas las & $58 \%$ frente a 12\% & $<0,0001$ & 4,91 \\
\hline
\end{tabular}

\section{Consumo de drogas}

Los adolescentes de nuestra encuesta que han consumido alcohol, tabaco y otras drogas también han sido más frecuentemente precoces en cuanto al sexo (Tabla 5).

\section{Otras relaciones encontradas}

La memoria autopercibida, la calidad de su alimentación o de su salud actual también se relacionan de forma estadísticamente significativa con tener o no relaciones sexuales completas (Tabla 4). Sin embargo solo encontramos diferencias en ciertos tramos de edad con lo que debemos ser cautos a la hora de inferir conclusiones.

En el caso de la religión, encontramos que, a los 13 años, el $52 \%$ de los que han tenido relaciones sexuales se definen como agnósticos o ateos frente a un $22 \%$ de católicos no practicantes y un $26 \%$ de católicos no muy practicantes. Sin embargo, los de su misma edad sin relaciones sexuales completas se definen en un $19 \%$ como agnósticos o ateos, en un $28 \%$ como católicos no practicantes, en otro $28 \%$ como católicos no muy practicantes, en un $23 \%$ como católicos practicantes y en un $2 \%$ como creyentes de otra religión. Las diferencias son estadisticamente significativas $(p=0,01)$.

\section{Factores que no aumentan o que no se relacionan} con la precocidad sexual

También en nuestra encuesta preguntábamos sobre otras situaciones en las que no hemos visto diferencias significativas entre los que han tenido relaciones sexuales completas y los que no. Serían la pertenencia a un grupo $(p=0,97)$, el nivel de información sobre el efecto de las drogas $(p=0,13)$, hacer deporte $(p=0,19)$, la participación en el tema sexualidad en su centro $(p=0,26)$, creer que embarazarse es un riesgo para los adolescentes $(p=0,07)$, la orientación sexual $(p=0,24)$ y la salud infantil $(p=0,31)$.

Además existen otros ítems que en un principio parecían relacionados con haber tenido relaciones sexuales completas, pero si ajustábamos por edad las diferencias no eran significativas $(p>0,05)$. Serían la información sobre ITS, sobre sexualidad, el estado de ánimo y la autoestima.

\section{DISCUSIÓN}

\section{Chico frente a chica}

En nuestro estudio, y en casi todos los estudios sobre sexualidad en adolescentes, se compara cada 


\begin{tabular}{|c|c|c|c|c|}
\hline \multirow{2}{*}{$\begin{array}{l}\text { Creencia } \\
\text { Edad que creen mejor para hablar de sexualidad: } \\
\text { - Antes de } 5 \text { años } \\
\text { - 5-8 años } \\
\text { - } 9 \text { a } 11 \text { años } \\
\text { - } 12 \text { a } 14 \text { años } \\
\text { - Nunca }\end{array}$} & \multicolumn{2}{|c|}{$\begin{array}{c}13 \text { años } \\
\text { Relaciones sexuales: sí/no } \\
(p<0,05)\end{array}$} & \multicolumn{2}{|c|}{$\begin{array}{c}14 \text { años } \\
\text { Relaciones sexuales: sí/no } \\
(p<0,05)\end{array}$} \\
\hline & $\begin{array}{c}\text { Sí } \\
13 \% \\
13 \% \\
30 \% \\
43 \% \\
- \\
\end{array}$ & $\begin{array}{c}\text { No } \\
0,3 \% \\
4 \% \\
18 \% \\
74 \% \\
4 \%\end{array}$ & $\begin{array}{c}\text { Sí } \\
9 \% \\
7 \% \\
25 \% \\
56 \% \\
2 \% \\
\end{array}$ & $\begin{array}{c}\text { No } \\
0,9 \% \\
1,3 \% \\
20 \% \\
77 \% \\
0,9 \% \\
\end{array}$ \\
\hline $\begin{array}{l}\text { Creen que lo que saben de sexualidad es: } \\
\text { - Muy insuficiente } \\
\text { - Insuficiente } \\
\text { - Suficiente } \\
\text { - Más que suficiente }\end{array}$ & $\begin{array}{c}\text { Sí } \\
- \\
9 \% \\
43 \% \\
48 \%\end{array}$ & $\begin{array}{l}\text { No } \\
3 \% \\
13 \% \\
65 \% \\
19 \%\end{array}$ & $\begin{array}{c}\text { Sí } \\
4 \% \\
5 \% \\
62 \% \\
29 \%\end{array}$ & $\begin{array}{l}\text { No } \\
2 \% \\
14 \% \\
70 \% \\
14 \%\end{array}$ \\
\hline
\end{tabular}

respuesta en función del sexo del encuestado. Nuestros resultados afirman que los chicos son más precoces que las chicas. Este hecho también se ve reflejado en diferentes trabajos anteriores ${ }^{3,7}$. Además, los varones suelen tener más parejas ocasionales y adoptan mayores riesgos que las chicas. En cambio, las chicas suelen tener relaciones sexuales en el marco de una relación estable y asociada a una relación de amor y confianza ${ }^{7}$.

$Y$ es que chicos y chicas viven la sexualidad de modo esencialmente diferente, a pesar de que en los últimos años parecen haberse diluido algo esas diferencias. Las mujeres asocian la actividad sexual más a los afectos, la intimidad y el compromiso que los varones. De hecho, suelen tener menos parejas y dar un significado afectivo y social más fuerte a la pareja que han tenido o tienen. Otro hecho diferencial es que los chicos presentan una mayor frecuencia de fantasías sexuales y mayor deseo sexual que las chicas y son más activos en la búsqueda de relaciones ${ }^{8,9}$.

Estas diferencias en motivación, exclusividad, atracción, deseo, fantasías e iniciativa reflejan un patrón bien evidente: los chicos tienen motivaciones sexuales más explícitas y más abiertas a diferentes personas, a la vez que la sexualidad ocupa más su mente y sus afectos sexuales; mientras las chicas vinculan más la sexualidad a los afectos y a una pareja o un número más reducido de personas, a la vez que piensan menos explícitamente en la sexualidad. Aun así, este cuadro deja fuera a un número relevante de adolescentes, por lo que parece tratarse de un patrón en proceso de cambio. Proceso que aparece especialmente claro por los cambios que se dan en relación con la edad, acercando en general las chicas a los chicos a medida que van cumpliendo años ${ }^{7,8}$.

Para explicar este doble patrón existen razones biológicas, evolucionistas y culturales, según López et al. Según este mismo autor, el cambio más importante que se ha dado en la sociedad actual en los últimos años es que actualmente las mujeres pueden tomar la iniciativa en las relaciones sexuales, pueden decir libremente "sí o no" y han dejado de cumplir con la función asignada en las sociedades tradicionales: "decir no a los varones, si no era en el matrimonio heterosexual y con el fin de tener hijos"8.

\section{Alcohol y otras drogas}

Uno de los factores de riesgo de precocidad sexual más ampliamente estudiado es el consumo de alcohol y otras drogas. En los ambientes recreativos nocturnos diferentes estudios muestran que, cuando hay perspectiva de encuentros sexuales, se puede derivar hacia un mayor consumo de drogas. Son muchos los jóvenes que valoran las sustancias (muy en especial el alcohol, pero también otras como cannabis, cocaína o éxtasis) por sus efectos facilitadores de logros sexuales que, abarcan un amplio abanico de objetivos que van desde la 
relajación hasta una completa desinhibición. En las entrevistas a jóvenes de un estudio reciente se admitía que la mejor forma de ligar es ir directamente a ciertos locales en los que se sabe que es habitual que quienes acuden estén bajo la influencia del alcohol o las drogas ${ }^{10,11}$.

El alcohol es la sustancia preferida por los jóvenes porque facilita el encuentro sexual, involucrarse en experiencias más arriesgadas y aumenta la excitación. La segunda sustancia más valorada es la cocaína porque permite prolongar la relación sexual. Pero también encontramos consecuencias negativas derivadas de la asociación entre consumo de drogas y relaciones sexuales. Esto es debido a la disminución de la percepción de riesgo que producen estas sustancias, lo que hace más probable mantener relaciones sexuales sin protección, lo cual incrementa los embarazos no deseados y las enfermedades de transmisión sexual ${ }^{12}$. Asimismo las drogas se asocian a experiencias sexuales efímeras como "ligues de una sola noche"13.

El Instituto Nacional de Abuso de Alcohol y Alcoholismo de EE. UU. reporta que las personas con desórdenes de consumo de alcohol tienen mayor probabilidad de contraer infección por virus de la inmunodeficiencia humana (VIH) y otras enfermedades de transmisión sexual que la población general debido a relaciones sexuales sin protección, múltiples parejas, relaciones con trabajadoras sexuales y sexo a cambio de alcohol o drogas ${ }^{14}$.

Calafat et al. en dos estudios concluyeron que las mujeres son más conscientes que los hombres de los riesgos que supone tener relaciones sexuales bajo los efectos del alcohol, pero que esto no se traduce en acciones preventivas. Cabe subrayar la asociación que encontraron entre el consumo de alcohol y la práctica de conductas de riesgo, especialmente en las primeras experiencias sexuales ${ }^{10,11}$. En un estudio reciente, se puso de manifiesto que, a pesar de que las mujeres consumían menos drogas y tenían más conciencia de posibles problemas, no encontraba diferencias entre sexos ni en la utilización de preservativos, ni en adoptar medidas de control de natalidad, ni en haber practicado sexo del que luego se han arrepentido, pero sí en que los varones tenían más relaciones bajo los efectos del alcohol y de las drogas ${ }^{11}$.

Nuestros datos coinciden con otros estudios que señalan que el consumo de sustancias como el alcohol, cannabis, cocaína o éxtasis antes de los 16 años está relacionado con un inicio más temprano en las relaciones sexuales. Además, los consumidores de drogas en el ámbito de salir de marcha han mantenido relaciones sexuales con un mayor número de parejas e, incluso, es más probable que hayan pagado por mantener relaciones sexuales ${ }^{12}$. Por otro lado, se ha observado una relación entre el consumo de drogas y el uso de la anticoncepción de emergencia ${ }^{15}$

Es un hecho que el consumo de drogas relaja las costumbres sexuales y, en consecuencia, lleva a mantener relaciones descuidadas e inseguras. El $46,75 \%$ de los jóvenes de un estudio reciente reconocieron que las drogas y el alcohol habían influido en que mantuviesen relaciones sexuales inseguras. Sin embargo, en los 12 meses anteriores, el $8,2 \%$ mantuvieron relaciones de las que se arrepintieron posteriormente debido al consumo de sustancias, y el $24,5 \%$ se sometieron a pruebas de detección de ITS, lo cual demuestra la percepción del riesgo de adoptar estas conductas sexuales.

La relajación, la euforia, la desinhibición, la disminución del autocontrol y de la percepción del riesgo provocado por las sustancias psicoactivas llevan a los consumidores a ser menos cuidadosos y a que olviden los mensajes de sexo seguro que habrían puesto en práctica si estuviesen sobrios ${ }^{13}$.

Nuestros datos son concordantes con todo lo anterior: hay una clara la relación entre las conductas sexuales de los jóvenes y el consumo de alcohol y drogas. Así, los consumidores de alcohol y drogas tienden a iniciar su vida sexual antes, a tener más parejas sexuales y a adoptar mayor número de conductas sexuales potencialmente peligrosas en comparación con los no consumidores ${ }^{13}$.

\section{Disfunción familiar}

En nuestro estudio se comprueba que los hijos que tienen peor relación con sus padres y también los 
de 14 años con padres divorciados son más precoces en sus relaciones sexuales. De acuerdo a lo reportado en la literatura médica internacional, la crianza por ambos padres y los hijos que viven con ambos padres experimentan menos actividad sexual e inician actividad sexual a edades mayores. Por el contrario, la mala relación con los padres, la mala comunicación familiar, los padres con antecedentes de paternidad o maternidad adolescente, en definitiva, la disfunción familiar está relacionada con el inicio sexual antes de los 15 años ${ }^{16}$.

Además se ha demostrado que la falta de supervisión de los padres y de comunicación padres-hijos o el grado de a probación de las relaciones sexuales de los hijos por parte de los padres son importantes determinantes de sexualidad precoz.

En un estudio reciente se encontró un porcentaje estadísticamente mayor de jóvenes con relaciones sexuales entre los que no utilizaban como fuente de información a los padres. En estos resultados se observa que la frecuencia de haber tenido relaciones sexuales era menor en aquellos que frecuentemente habían tratado con los padres cuestiones sobre el amor y el enamoramiento, así como sobre temas de biología sexual, como los cambios sexuales que experimentan ${ }^{17}$.

Por tanto, aparece como prioritario incluir a los padres en cualquier programa de prevención del embarazo adolescente ${ }^{16}$.

\section{Creen que lo que saben de sexualidad es más que suficiente}

Los adolescentes de 13 y 14 años con relaciones sexuales respecto a los que no han tenido refieren que lo saben de sexualidad es más que suficiente. Este hecho contrasta con diferentes estudios que refieren que un mayor nivel de conocimientos sobre las ITS y la prevención de embarazos no deseados están relacionado con iniciar las relaciones sexuales más tarde.

El que crean que lo que saben de sexualidad es más que suficiente es un dato preocupante. Diferentes estudios señalan que los adolescentes no tienen en general un conocimiento alto sobre sexualidad ${ }^{18}$ y esa arrogancia de los sexualmente activos nos hace temer un aumento de las ITS y de los embarazos no deseados.

\section{Menos religiosos}

En nuestro estudio hemos observado que existen diferencias estadísticamente significativas en la precocidad sexual en los adolescentes de 13 años que se consideran no religiosos, agnósticos o ateos. Parece pues que un mayor acercamiento a la religión católica se asocia a un retraso en el inicio sexual. Este hecho se ha comprobado también en otros estudios ${ }^{17,19}$. Según otros autores, incluso la religiosidad parental se asocia significativamente a la postergación del inicio sexual en hombres y mujeres ${ }^{16}$.

\section{Más erotofílicos}

La erotofilia se asocia positivamente a una mayor frecuencia de actividad autoerótica, más experiencias sexuales en el pasado y mayor número de parejas sexuales. Es normal que, de forma estadísticamente significativa, los adolescentes más erotofílicos sean los que hayan debutado sexualmente ${ }^{20}$. Lo positivo es que, según la literatura científica, las personas más erotofílicas prestan más atención, procesan y recuerdan más información sexual, anticipan la posibilidad de tener relaciones sexuales y adquieren en mayor medida métodos anticonceptivos, siendo estas habilidades eficaces para la prevención de conductas de riesgo ${ }^{7}$.

\section{CONCLUSIONES}

La precocidad sexual de nuestros adolescentes está fuertemente relacionada con el consumo de drogas, de alcohol y de tabaco. También se haya relacionado con el hecho de ser más erotofílicos y de ser chico.

Además los adolescentes que han tenido relaciones sexuales creen que saben mucho (incluso más que suficiente) sobre sexualidad, lo que nos hace temer que tomen aún menos precauciones. 
Otro de los factores claramente relacionados con la precocidad sexual es tener una peor relación con sus padres. Puede que aquí sea donde pediatras, pedagogos, autoridades y sobre todo padres tengamos una oportunidad para favorecer una sexualidad sana en nuestros adolescentes. Tarea difícil, pero la alternativa parece mucho peor.

\section{CONFLICTO DE INTERESES}

Los autores declaran no presentar conflictos de intereses en relación con la preparación y publicación de este artículo.

\section{BIBLIOGRAFÍA}

1. Resultados de la Encuesta Nacional de Salud Sexual 2009. Ministerio de Sanidad y Política Social [en línea] [consultado el 12/06/2015]. Disponible en www. msps.es/organizacion/sns/planCalidadSNS/docs/ v5_presentacion_ResultadosENSS_16dic09.pdf

2. Calatrava M, López-Del Burgo C, de Irala J. Factores de riesgo relacionados con la salud sexual en los jóvenes europeos. Med Clin (Barc). 2012;138:534-40.

3. Rodríguez-Carrión J, Traverso Blanco Cl. Conductas sexuales en adolescentes de 12 a 17 años de Andalucía. Gac Sanit. 2012;26:519-24.

4. Junta de Castilla y León. Portal de Educación [en línea] [consultado 01/07/2012]. Disponible en www. educa.jcyl.es/es/informacion/estadistica-ensenan za-universitaria/curso-2010-2011

5. Casal J, Mateu E. Tipos de muestreo. Rev Epidemiol Med Prev. 2003;1:3-7.

6. Pérez López C. Muestreo estadístico. Conceptos y problemas resueltos. Madrid: Pearson Educación; 2005.

7. García-Vega E, Menéndez Robledo E, García Fernández P, Rico Fernández R. Influencia del sexo y del género en el comportamiento sexual de una población adolescente. Psicothema. 2010;22:606-12.

8. López F, Carcedo R, Fernández Rouco N, Blázquez MI, Kilani A. Diferencias sexuales en la sexualidad adolescente: afectos y conductas. An Psicología. 2011; 27:791-9.

\section{FINANCIACIÓN}

El Departamento de Pediatría de la Universidad de Valladolid financió la grabación de datos de este trabajo a cargo de Ágora Estudios de Mercado.

\section{ABREVIATURAS}

ESO: Educación Secundaria Obligatoria • ITS: infección de transmisión sexual • VIH: virus de la inmunodeficiencia humana.

9. Bermúdez MP, Teva I, Buela-Casal G. Influencia de variables sociodemográficas sobre los estilos de afrontamiento, el estrés social y la búsqueda de sensaciones sexuales en adolescentes. Psicothema. 2009;21: 220-6.

10. Calafat A, Juan M, Becoña E, Mantecón A. Qué drogas se prefieren para las relaciones sexuales en contextos recreativos. Adicciones. 2008;20:37-48.

11. Calafat A, Juan M, Becoña E, Mantecón A, Ramón A. Sexualidad de riesgo y consumo de drogas en el contexto recreativo. Una perspectiva de género. Psicothema. 2009;21:227-33.

12. Becoña Iglesias E, López-Durán A, Fernández del Río E, Martínez Pradeda U, Osorio López J, Fraga Ares J, et al. Borracheras, conducción de vehículos y relaciones sexuales en jóvenes consumidores de cocaína y éxtasis. Adicciones. 2011;23:205-18.

13. Lomba L, Apóstolo J, Mendes F. Consumo de drogas, alcohol y conductas sexuales en los ambientes recreativos nocturnos de Portugal. Adicciones. 2009; 21:309-26.

14. Castaño Pérez GA, Arango Tobon E, Morales Mesa S, Rodríguez Bustamante A, Montoya Montoya C. Consumo de drogas y prácticas sexuales de los adolescentes de la ciudad de Medellin (Colombia). Adicciones. 2012;24:347-54

15. López-Amorós M, Schiaffino A, Moncada A, Pérez G. Factores asociados al uso autodeclarado de la anticoncepción de emergencia en la población escolarizada de 14 a 18 años de edad. Gac Sanit. 2010;24: 404-9. 
16. González E, Molina T, Montero A, Martínez V. Factores familiares asociados al inicio sexual temprano en adolescentes consultantes en un centro de salud sexual y reproductiva en Santiago de Chile. Rev Med Chile. 2013;141:313-9.

17. Ruiz-Canela M, López-del Burgo C, Carlos S, Calatrava M, Osorio A, de Irala J. Familia, amigos y otras fuentes de información asociadas al inicio de las relaciones sexuales en adolescentes de El Salvador. Rev Panam Salud Pública. 2012;31:54-61.

18. Espada JP. Validación de una escala de conocimiento sobre el VIH y otras infecciones de transmisión sexual en población adolescente. Aten Primaria. 2014; $46: 558-65$
19. Sierra JC, Vallejo-Medina P, Santos-Iglesias P, Lameiras Fernández M. Validación del Massachusetts General Hospital-Sexual Functioning Questionnaire (MGH-SFO) en población española. Aten Primaria. 2012;44:516-26.

20. Carpintero E, Fuertes A. Validación de la versión en español del "Sexual Opinion Survey" (SOS). Cuadernos de Medicina Psicosomática. 1994;31:52-61. 